Published in final edited form as:

Langmuir. 2005 December 20; 21(26): 12124-12129. doi:10.1021/la052590q.

\title{
Shrink-wrap Vesicles
}

\author{
Shelly M. Fujikawa, Irene A. Chen, and Jack W. Szostak ${ }^{*}$ \\ Howard Hughes Medical Institute, and Dept. of Molecular Biology, Massachusetts General Hospital, \\ Boston, MA 02114
}

\section{Abstract}

We describe a simple approach to the controlled removal of molecules from the membrane of large unilamellar vesicles made of fatty acids. Such vesicles shrink dramatically upon mixing with micelles composed of a mixture of fatty acid and phospholipid (POPC), as fatty acid molecules leave the vesicle membrane and accumulate within the mixed micelles. Vesicle shrinkage was confirmed by dynamic light scattering, fluorescence recovery after photobleaching of labeled vesicles, and fluorescence resonance energy transfer between lipid dyes incorporated into the vesicle membrane. Most of the encapsulated impermeable solute is retained during shrinkage, becoming concentrated by a factor of at least 50 -fold in the final small vesicles. This unprecedented combination of vesicle shrinkage with retention of contents allows for the preparation of small vesicles containing high solute concentrations, and may find applications in liposomal drug delivery.

\section{Introduction}

Vesicles are of interest as agents of drug delivery because they reduce the toxicity and improve the pharmacokinetics of certain drugs, while providing a protective barrier for entrapped macromolecules $1-3$. Nanosized vesicles are of special interest because the efficiency of delivery generally increases as particle size decreases $1,4-6$. Small vesicles may be able to penetrate small pores (e.g., endothelial defects) and also exhibit increased circulation times, and these features can lead to greater accumulation in the hypervascularized tissue of solid tumors $4,6-8$. The smallest vesicles currently used are still relatively large $(\sim 100 \mathrm{~nm})$, so vesicles of even smaller size may have additional utility. Small particles could also be useful for inhaled drugs, having an increased probability of deposition as well as an enhanced ability to cross the pulmonary epithelium 9,10 .

The amount of drug encapsulated by small vesicles is usually quite low, since the encapsulated volume decreases with the third power of the radius. Here we describe a method for preparing small vesicles (outer diameter 20-25 nm) with highly concentrated internal contents, by first preparing large unilamellar vesicles (outer diameter $\sim 70 \mathrm{~nm}$ ) from myristoleic acid (MA) and then shrinking these membranes in a process that retains the majority of encapsulated dye molecules.

Vesicle shrinking is a surprising phenomenon that is a consequence of the dynamic behavior of single-chain amphiphiles in bilayer membranes. Fatty acid vesicles can incorporate

szostak@molbio.mgh.harvard.edu Tel: 617-726-5981. Fax: 617-726-6893.

Supporting Information Available: Standard curve of donor fluorescence vs. probe density for the FRET assay (Fig. S1). Standard curve of self-quenching of calcein vs. concentration (Fig. S2). Release of calcein during and after shrinking of MA vesicles (Fig. S3). Final vesicle diameter after shrinking using micelles composed of varying mole percentages of POPC and MA (Fig. S4). Fluorescence of encapsulated calcein after the addition of hypertonic solution of bicine (Fig. S5). Shrinking of oleate vesicles after adding MA/POPC (89\% MA, 11\% POPC) micelles (Fig. S6). Supplementary text and references. This material is available free of charge via the Internet at http://pubs.acs.org. 
additional fatty acid (supplied as micelles) into their membranes and thereby grow larger

11-13. We have previously observed both the growth of osmotically swollen vesicles and a small degree of shrinkage of osmotically relaxed vesicles following the mixing of these vesicles, as fatty acid molecules are transferred from the relaxed to the swollen vesicles ${ }^{14}$. Vesicle growth proceeds through incorporation of fatty acid into the outer leaflet of the membrane, followed by flip-flop of approximately half of these molecules into the inner leaflet 15. The flip-flop of fatty acid molecules is much faster than that of most phospholipids, such as POPC (1-palmitoyl-2-oleoyl-sn-glycero-3-phosphocholine $)^{16-19}$. We attempted to take advantage of the slow flip-flop of phospholipids in an effort to generate asymmetric membranes with an excess of phospholipid in the outer leaflet, by mixing MA vesicles with MA micelles doped with POPC (11 mol\% POPC; 89 mol\% MA).

However, instead of incorporating into the vesicles, the mixed myristoleic acid/phospholipid micelles extracted fatty acid from the vesicles. Most of the initially encapsulated dye remained entrapped in the vesicles after shrinkage was complete. This yielded very small vesicles with highly concentrated contents (50-100-fold increase in concentration).

\section{Methods and Materials}

\section{Vesicle preparation}

Vesicles were prepared as described ${ }^{13}$. Briefly, myristoleic acid (Nu-Chek Prep, Inc., Elysian, $\mathrm{MN}$ ) was mixed with one equivalent of $\mathrm{NaOH}$ in water, followed by addition of $1 \mathrm{M}$ bicine to obtain a visibly turbid solution of $100 \mathrm{mM}$ MA in $0.2 \mathrm{M}$ bicine, $\mathrm{pH}$ 8.5. After extrusion through $0.1 \mu \mathrm{m}$ polycarbonate filters using a Mini-extruder (Avanti Polar Lipids, Alabaster, AL), vesicles were incubated overnight for equilibration. All subsequent vesicle dilutions were done in $4 \mathrm{mM}$ MA, $0.2 \mathrm{M}$ bicine, $\mathrm{pH} 8.5 .4 \mathrm{mM}$ is approximately equal to the critical aggregate concentration (cac) of MA and was necessary to preserve membrane integrity after dilution.

For the FRET experiments, vesicles were labeled with 1,2-dihexadecanoyl-sn-glycero-3phosphoethanolamine (Rh-DHPE) and $N$-(7-nitrobenz-2-oxa-1,3-diazol- 4-yl)-1,2dihexadecanoyl-sn-glycero-3-phosphoethanolamine (NBD-PE) (Molecular Probes, Eugene, $\mathrm{OR}$ ). Vesicles contained $0.025 \mathrm{~mol} \%$ of each dye and were prepared as described (Hanczyc et al., 2003). The standard curve was prepared using 0.025-0.4 mol\% of each dye.

To prepare vesicles encapsulating calcein or HPTS (Molecular Probes, Eugene, OR), 0.2-20 $\mathrm{mM}$ calcein or $0.5 \mathrm{mM}$ HPTS was mixed with bicine before addition to MA micelles. Extruded vesicles were purified from unencapsulated dye by size-exclusion chromatography on Sepharose 4B (Sigma-Aldrich, St. Louis, MO).

For FRAP experiments, vesicles were labeled with $0.5 \%$ NBD-PE. At this concentration of NBD-PE, vesicle formation from micelles resulted in relatively small vesicles, as determined by DLS. Instead, these vesicles were prepared by resuspending the oil in a solution of $0.2 \mathrm{M}$ bicine for a final $\mathrm{pH}$ of $8.5^{15}$.

The concentration of MA vesicles in each preparation was determined using the ADIFAB fatty acid assay (Molecular Probes) 20 .

\section{MA/POPC micelle preparation}

MA/POPC micelles were prepared by combining solutions of MA in methanol and POPC (1palmitoyl-2-oleoyl-sn-glycero-3-phosphocholine; Avanti Polar Lipids, Alabaster, AL) in chloroform at an 89:11 mol/mol ratio, unless otherwise specified. Volatile solvents were removed by rotary evaporation. The resulting oil was dissolved in water with one equivalent of $\mathrm{NaOH}$ to yield a clear solution with a final total lipid concentration of $50 \mathrm{mM}$ and $\mathrm{pH}>10$. 
For FRAP experiments with labeled micelles, $0.5 \mathrm{~mol} \%$ NBD-PE was added to the mixture prior to rotary evaporation.

\section{Micelle addition to vesicles}

Shrinking reactions were initiated by simultaneously adding MA/POPC micelles and $1 \mathrm{M}$ bicine, $\mathrm{pH} 8.5$, to MA vesicles in $0.2 \mathrm{M}$ bicine, $\mathrm{pH}$ 8.5. The added volume of $1 \mathrm{M}$ bicine, $\mathrm{pH}$ 8.5 , ensured that the final solution contained $0.2 \mathrm{M}$ bicine, $\mathrm{pH}$ 8.5. Reactions were mixed by pipetting and vortexed for $\sim 3$ seconds. Control mixtures contained MA vesicles diluted into buffer. In general, shrinking reactions contained $3.2 \mathrm{mM}$ vesicles and $4 \mathrm{mM}$ MA/POPC micelles unless otherwise specified. To determine the dependence of rate on micelle concentration, $3.2 \mathrm{mM}$ vesicles were mixed with $4-30 \mathrm{mM}$ micelles. To determine the dependence of rate on vesicle concentration, $4 \mathrm{mM}$ micelles were mixed with 0.42-3.2 mM vesicles. To attain acceptable signal strength, FRAP experiments contained $32 \mathrm{mM}$ vesicles and $20 \mathrm{mM}$ micelles. Reaction kinetics were fit to a first-order equation $\left(y=y_{0}+a * \exp (-k x)\right)$ to determine the rate constant $(k)$ and the final diameter $\left(y_{0}\right)$.

\section{Dynamic light scattering (DLS) and turbidity measurements}

DLS measurements of vesicles and shrinking reactions were performed using a PDDLS Coolbatch/PD2000DLS instrument at $25^{\circ} \mathrm{C}$ (Precision Detectors, Inc., Franklin, MA). This instrument is insensitive to very small particles $(<5 \mathrm{~nm})$ due to the lower limit on the correlation times that it can measure. Also, because of the strong dependence of scattering intensity on particle size, DLS measurement of shrinking reactions effectively measured the progress of changes in vesicle size. For the measurement of small micelles in the absence of vesicles, DLS was performed using a similar instrument capable of detecting shorter correlation times, the PDDLS Coolbatch 90T/PD2000DLS ${ }^{\text {Plus }}$ (Precision Detectors, Inc. Franklin, MA). Turbidity was measured by absorbance at $500 \mathrm{~nm}$ using a Cary $1 \mathrm{E}$ UV-Visible spectrophotometer (Varian, Inc., Walnut Creek, CA).

\section{Fluorescence resonance energy transfer (FRET) assay}

Shrinking reactions of labeled vesicles and MA/POPC micelles were measured over time in a quartz cuvette using a Cary Eclipse fluorimeter (Varian Inc., Walnut Creek, CA). The FRET signal was determined from the extent of quenching of donor fluorescence (NBD-PE; ex. 430 $\mathrm{nm}$, em. $530 \mathrm{~nm}$ ), as described 15,21,22. Vesicles were disrupted using Triton X-100 (1\%) to measure the unquenched fluorescence of the donor. A standard curve for FRET signal vs. probe density was used to translate measured fluorescence into probe density (Fig. S1) and therefore relative surface area (1/relative probe density). The corresponding relative diameter was calculated as the square root of the relative surface area. The relative diameter was converted to $\mathrm{nm}$ units by multiplying by the initial diameter of the vesicles, determined by DLS. The presence of the FRET dyes did not affect the rate of shrinking as determined by DLS.

\section{Calcein self-quenching and percent encapsulation}

Aliquots from a shrinking reaction were run on a Sephadex G-25 size-exclusion column to separate vesicles with encapsulated calcein from free calcein. To determine the internal concentration of calcein in the vesicles, the fluorescence (ex. $415 \mathrm{~nm}, \mathrm{em} .600 \mathrm{~nm}$ ) of the vesicle fraction was measured before $\left(F_{v e s}\right)$ and after $\left(F_{\text {ves }}^{0}\right)$ addition of $1 \%$ Triton X-100. ${ }^{13}$ The extent of quenching $\left(F_{\text {ves }} / F^{0}{ }_{\text {ves }}\right)$ was related to calcein concentration by a standard curve (Fig. S2). The corresponding relative diameter was calculated as the (concentration factor) ${ }^{-1 / 3}$, after adjusting for dye leakage. The relative diameter was converted to nm units by multiplying by the initial diameter of the vesicles, determined by DLS. 
The percent dye encapsulated was determined as $F^{0}{ }_{\text {ves }} /\left(F^{0}{ }_{\text {ves }}+F_{\text {free }}\right)$, adjusted for the volumes of the fractions. For calcein, because dye release occurred while the vesicle size was decreasing as well as after the vesicle size had equilibrated, the percent dye encapsulated at the end of shrinking was determined by fitting a single exponential decay curve to time points collected in $<1000$ minutes (i.e. $<5$ half-lives) (for an example, see Fig. S3). For HPTS, the end of the shrinking reaction was defined to be $\sim 4$ half-lives, as determined by the decrease in vesicle size measured by DLS.

\section{Fluorescence recovery after photobleaching (FRAP) assay}

Vesicles and micelles were labeled with $0.5 \mathrm{~mol} \%$ NBD-PE in separate experiments. Shrinking reactions were incubated at room temperature, transferred to a glass slide, covered with a cover slip and sealed with clear nail polish. The diffusion constant of the labeled species was determined by FRAP, ${ }^{23}$ using a Leica TCS SP confocal laser scanning microscope (20x objective, $\mathrm{NA}=0.7$ ). A selected point was photobleached using the $488 \mathrm{~nm}$ argon laser line at $100 \%$ intensity for one second. The initial width and extent of bleaching were determined from a sample of labeled micelles that were dried onto a slide. Recovery data were collected at $11 \%$ intensity and successive frames were $600 \mathrm{~ms}$ apart ( $4 \mathrm{x}$ zoom, pinhole $=222 \mu \mathrm{m}$ ). Data were normalized by total image intensity. The characteristic diffusion time was determined by fitting to the series solution for 2-D diffusion with a Gaussian beam profile, truncated after 40 terms, to calculate the diffusion constant (D). ${ }^{24}$ The diameter was calculated according to Stokes' law, assuming spherical particles $(\mathrm{D}=\mathrm{kT} /(6 \pi \eta r))$. Results were averaged from at least five replicates. The presence of $0.5 \%$ NBD-PE did not affect the rate of shrinking determined by DLS.

\section{Results and Discussion}

We expected that the incorporation of molecules derived from the MA/POPC micelles into the vesicle membrane would cause an increase in average particle size, as seen for vesicle growth following the addition of MA micelles ${ }^{13}$. However, in initial experiments we observed a decrease, instead of the expected increase, in turbidity (Fig. 1). Because changes in turbidity may reflect changes in particle size, shape, or number, we also measured the diffusion constant of the particles by dynamic light scattering (DLS). DLS indicated that the mean hydrodynamic diameter $\left(d_{\mathrm{H}}\right)$ of the vesicles decreased from $73 \mathrm{~nm}$ to $20 \mathrm{~nm}$, in an apparent first-order process $\left(k_{\text {DLS }} \sim 0.004 \mathrm{~min}^{-1}\right)$ (Table 1; Fig. 2A). This decrease was also observed using micelles of different POPC content (5.5-16.5 mol\%) (Fig. S4).

Two processes could lead to a decrease in average vesicle size: division or shrinking. To distinguish between these possibilities, we prepared MA vesicles containing a pair of fluorescent phospholipid dyes incorporated into the membrane. These dyes form a fluorescence resonance energy transfer (FRET) pair and do not exchange detectably among vesicles for many hours $13,14,22$. The efficiency of FRET depends strongly on the distance between dye molecules and thus measures the surface density of the dyes (Fig. S1) ${ }^{21}$. If the vesicles were dividing, the surface density of the dyes would not change. But if vesicles were shrinking by extraction of MA out of the membranes, the surface density of the dyes would increase. We found that the surface density of the dyes increased upon mixing labeled vesicles and MA/ POPC micelles. After translating these data into relative diameter (assuming the vesicles were spherically shaped), the decrease of vesicle size over time measured by the FRET assay was similar to that measured by DLS ( $36 \pm 1 \%$ of the initial diameter; Table 1; Fig. $2 B$ ). This result indicated that the vesicles were shrinking, not dividing.

An important question was whether the internal contents of the vesicles were concentrated during shrinking, rather than being released into bulk solution. We encapsulated a watersoluble, self-quenching fluorescent dye (calcein) in MA vesicles. As shrinking progressed, 
aliquots of the reaction were purified by size-exclusion chromatography to separate the vesicles, containing entrapped calcein, from calcein that had leaked into free solution. Although there was some leakage, most of the initially encapsulated calcein copurified with the vesicle fraction (Fig. S3), and this percentage was similar for different concentrations of initially encapsulated calcein. For vesicles initially encapsulating $20 \mathrm{mM}$ calcein, $58 \pm 6 \%$ of the dye was retained after shrinking, and $52 \pm 13 \%$ of the dye was retained by vesicles initially encapsulating $2 \mathrm{mM}$ calcein. Another fluorescent dye, HPTS, showed greater retention at the end of the vesicle shrinking reaction $(\sim 80 \%)$ (Fig. 3). The shrinking reaction also increased the permeability of the membrane, leading to the slow release of dye after the vesicle size had equilibrated, a potentially interesting property for a delivery vehicle.

The self-quenching efficiency of calcein fluorescence in the vesicle fraction is a measurement of the internal concentration of calcein (Fig. S2) ${ }^{13,25}$. Although the dynamic range of selfquenching is limited ( 7-30 mM calcein), the effective dynamic range of the assay could be made quite large by preparing vesicles with different initial concentrations of calcein $\left(c_{0}=0.2\right.$ - $20 \mathrm{mM})$. The change in the inner diameter $\left(d_{\mathrm{i}}\right)$ of the vesicles as a function of time was determined by compiling self-quenching data from several experiments, such that later time points were taken from experiments containing lower $c_{0}$ (e.g., $0.2 \mathrm{mM}$ ).

Using this assay, we found that encapsulated calcein was concentrated by a factor of 50-100, from $0.2 \mathrm{mM}$ to $10-20 \mathrm{mM}$. The resulting shrinking time course closely resembled that determined by DLS and the FRET assay (Table 1; Fig. 2C). The relative decrease in $d_{\mathrm{i}}$ is expected to be greater than the relative decrease in hydrodynamic diameter, because of the non-zero thickness of the membrane. For an estimated membrane thickness of $4 \mathrm{~nm} 26,27$, the decrease of $d_{\mathrm{H}}$ from $73 \mathrm{~nm}$ to $20 \mathrm{~nm}$, measured by DLS, would correspond to a decrease of $d_{\mathrm{i}}$ from $65 \mathrm{~nm}$ to $12 \mathrm{~nm}$, or $18 \%$ of the initial $d_{\mathrm{i}}$. From the calcein quenching experiments, we observed a final relative encapsulated diameter of $19 \%$, which agrees well with the value expected based on the DLS data.

While these data confirmed that the encapsulated volume was shrinking, we wondered whether this degree of dye concentration was reasonable given the consequent rise in osmotic pressure. The maximum tolerable osmotic pressure gradient is given by Laplace's law for a sphere: $\tau^{*}$ $=\left(\Delta \Pi^{*}\right) r / 2$, where $\tau^{*}$ is the rupture tension of the membrane, $\Delta \Pi^{*}$ is the maximum sustainable osmotic pressure gradient, and $r$ is the radius of the sphere. ${ }^{28,29}$ For $\tau^{*} \sim 10 \mathrm{dyn} / \mathrm{cm}^{14}$, the maximum gradient for a sphere of inner diameter $=12 \mathrm{~nm}$ would be $\sim 0.7$ Osm. Given the observed 50-100-fold increase in concentration of calcein, for $c_{0}=2 \mathrm{mM}(\sim 10 \mathrm{mOsm}$ due to the negative charges on calcein), the final internal osmolarity would be $\sim 0.5-1$ Osm after shrinking, close to the calculated maximum gradient. The osmotic contribution of the buffer (bicine) could be ignored because it equilibrates across the membrane within the timescale of the experiments (Fig. S5). However, the same calculation for $c_{0}=20 \mathrm{mM}$ would imply an osmotic pressure gradient greater than $\Delta \Pi^{*}$. Since the final degree of dye concentration was determined from measurements at low $c_{0}$, we tested whether vesicles with $c_{0}=20 \mathrm{mM}$ shrank less than vesicles with $c_{0} \leq 2 \mathrm{mM}$ calcein. Indeed, DLS measurements showed that vesicles with $c_{0}=20 \mathrm{mM}$ shrank less (Table 1), suggesting that the increased internal osmotic pressure inhibited further shrinking.

We also investigated the fate of the MA/POPC micelles after mixing with MA vesicles. Because spherical micelles are quite small, they scatter little light compared to vesicles, such that DLS primarily monitors the vesicles in the reaction. However, micelles can be labeled with a non-exchanging fluorescent phospholipid ( $N$-(7-nitrobenz-2-oxa-1,3-diazol-4-yl)-1,2dihexadecanoyl-sn-glycero-3-phosphoethanolamine; NBD-PE), and the size of these particles can be monitored by fluorescence recovery after photobleaching (FRAP) ${ }^{30}$. In FRAP, a sample is placed on a microscope slide and a small region is photobleached by a high intensity 
laser pulse. The recovery of fluorescence into this region measures the diffusion constant, and thus the size, of the fluorescent particles 23,31 . If labeled MA/POPC micelles are mixed with buffer at $\mathrm{pH} 8.5$, they show only a slight increase in size (Fig. 2D), whereas labeled MA micelles show a dramatic increase in size as they aggregate into large vesicular structures visible under a light microscope (up to several microns) ${ }^{13}$. This indicates that adding POPC to MA micelles inhibits the transition from micelles to a vesicular phase, consistent with the observed lack of incorporation of these micelles into preformed vesicles.

When labeled MA/POPC micelles are mixed with vesicles, the micelles show a slight increase in size (Fig. 2D), as expected. If all of the fatty acid extracted from the vesicles had been incorporated into the micelles, the diameter of the micelles would have increased by only $36 \%$. For particles of this small size, a change in micelle diameter of this magnitude is difficult to measure accurately by FRAP, but our data are consistent with this possibility. These results do indicate that MA/POPC micelles remain as small particles throughout the vesicle shrinking reaction.

In a separate FRAP experiment, the vesicles were labeled and monitored after the addition of MA/POPC micelles, confirming that the vesicles decreased in size (Fig. 2E; Table 1). Because FRAP required a relatively high amount of fluorescence to attain an acceptable signal to noise ratio, a higher initial concentration of MA vesicles was used for these experiments.

Nevertheless, the results were similar to those obtained from the other techniques. Also, the sizes of the micelles and vesicles measured by FRAP were distinct from each other, indicating that NBD-PE did not exchange substantially between the two populations, consistent with the absence of exchange of the FRET dyes among vesicles.

By the DLS, FRET, calcein quenching, and FRAP assays, the shrinking of vesicles appeared to follow a first-order integrated rate law. For a true first order reaction, the initial rate of shrinking would be directly proportional to the initial vesicle concentration, resulting in a van't Hoff plot with slope $=1$. However, the initial rate of shrinking decreased with increasing vesicle concentration, indicating that vesicles inhibited the reaction (Fig. 4A). In contrast, increasing the concentration of micelles increased the reaction rate dramatically; in some cases, a 2-fold increase in the concentration of micelles resulted in a $\sim 20$-fold increase in initial rate (Fig. $4 B)$.

A possible explanation for these results is that MA/POPC micelles associate with MA vesicles soon after mixing, forming reactive complexes. Similar complexes have been implicated as intermediates in the growth of fatty acid vesicles 15 (supplementary text). The initial rate of transfer would depend on the structure of the complexes, such that more micelles per vesicle would lead to a greater rate of transfer. This accounts for the inhibition of the reaction by vesicles and the increase in reaction rate at higher micelle concentrations. Furthermore, the formation of complexes containing more micelles may lead to an apparent increase in the reaction order, consistent with the observed van't Hoff plot for micelles (Fig. 4B). The driving force for the decrease in vesicle size may be that the geometry of POPC, which usually prefers to form bilayer membranes, destabilizes spherical micelles. The uptake of MA into these micelles could result in the formation of bicelle-like structures with the exposed edges stabilized by fatty acid molecules (Fig. 4C) 32,33 . If the driving force is inherent to the structure of MA/POPC micelles, the shrinking reaction might be applied to other vesicle compositions. Indeed, oleic acid vesicles also shrink when mixed with MA/POPC micelles (Fig. S6). Vesicles made from a polymerizable fatty acid (tricosadiynoic acid) also shrink when mixed with MA/ POPC micelles, suggesting a possible way to stabilize the final small vesicles 34,35 . 


\section{Conclusion}

We have described an unprecedented phenomenon in which a gradual removal of molecules from a bilayer membrane vesicle leads to a decrease in vesicle size and a concomitant 'shrinkwrapping' of encapsulated impermeable contents. The vesicle contents become highly concentrated, by a factor of 50-100, reaching the maximum osmotic concentration allowed by the tensile strength of the membrane. Small vesicles can therefore be made to encapsulate large amounts of solutes despite their low volumes. For drug delivery applications, more work is needed to stabilize these vesicles to physiological conditions; one possibility is to polymerize the vesicles, perhaps by using a cross-linkable fatty acid or by encasing them in polymerizable shells $36-39$. This strategy for shrinking vesicles may then be useful for encapsulating substantial amounts of drugs in very small vesicles.

\section{Supplementary Material}

Refer to Web version on PubMed Central for supplementary material.

\section{Acknowledgements}

The authors thank M. M. Hanczyc for helpful discussions. JWS is an investigator of the Howard Hughes Medical Institute. SMF was supported by a National Science Foundation Graduate Research Fellowship. IAC was supported by the NIH Medical Scientist Training Program and an NIH Molecular Biophysics Training Grant. This work was supported in part by a grant from the NASA Exobiology Program (EXB03-0031-0018).

\section{References}

1. Allen, TM.; Stuart, DD. Liposomes: rational design. Janoff, AS., editor. Marcel Dekker, Inc.; New York: 1999. p. 63-87.

2. Campos, S.; Shapiro, CL. Liposomes: rational design. Janoff, AS., editor. Marcel Dekker, Inc.; New York: 1999. p. 363-377.

3. Fenske, DB.; Maurer, N.; Cullis, PR. Liposomes: a practical approach. second ed.. Weissig, V., editor. Oxford University Press; Oxford: 2003. p. 167-191.

4. Charrois GJ, Allen TM. Biochim. Biophys. Acta 2003;1609:102-108. [PubMed: 12507764]

5. Banerjee R. J. Biomater. Appl 2001;16:3-21. [PubMed: 11475357]

6. Simon BH, Ando HY, Gupta PK. J. Pharm. Sci 1995;84:1249-1253. [PubMed: 8801343]

7. Gabizon, A.; Barenholz, Y. Liposomes: rational design. Janoff, AS., editor. Marcel Dekker; New York: 1999. p. 343-377.

8. Matsumura Y, Maeda H. Cancer Res 1986;46:6387-6392. [PubMed: 2946403]

9. Taylor, KMG.; Farr, SJ. Liposomes in drug delivery. Patel, HM., editor. 2. Harwood Academic Publishers; Chur: 1993. p. 95-109.

10. Borm PJ, Kreyling W. J. Nanosci. Nanotechnol 2004;4:521-531. [PubMed: 15503438]

11. Berclaz N, Müller M, Walde P, Luisi PL. J. Phys. Chem. B 2001;105:1056-1064.

12. Blöchliger E, Blocher M, Walde P, Luisi PL. J. Phys. Chem. B 1998;102:10383-10390.

13. Hanczyc MM, Fujikawa SM, Szostak JW. Science 2003;302:618-622. [PubMed: 14576428]

14. Chen IA, Roberts RW, Szostak JW. Science 2004;305:1474-1476. [PubMed: 15353806]

15. Chen IA, Szostak JW. Biophys. J 2004;87:988-998. [PubMed: 15298905]

16. Chen IA, Szostak JW. Proc. Natl. Acad. Sci. U S A 2004;101:7965-7970. [PubMed: 15148394]

17. Kamp F, Zakim D, Zhang F, Noy N, Hamilton JA. Biochemistry 1995;34:11928-11937. [PubMed: 7547929]

18. Kornberg RD, McConnell HM. Biochemistry 1971;10:1111-1120. [PubMed: 4324203]

19. Hamilton JA. J. Lipid Res 1998;39:467-481. [PubMed: 9548581]

20. Richieri GV, Ogata RT, Kleinfeld AM. Mol. Cell Biochem 1999;192:87-94. [PubMed: 10331662]

21. Fung BK-K, Stryer L. Biochemistry 1978;17:5241-5248. [PubMed: 728398] 
22. Struck DK, Hoekstra D, Pagano RE. Biochemistry 1981;20:4093-4099. [PubMed: 7284312]

23. Axelrod D, Koppel DE, Schlessinger J, Elson E, Webb WW. Biophys. J 1976;16:1055-1069. [PubMed: 786399]

24. Phair RD, Misteli T. Nature 2000;404:604-609. [PubMed: 10766243]

25. Allen TM, Cleland LG. Biochim. Biophys. Acta 1980;597:418-426. [PubMed: 7370258]

26. A single molecule of myristoleic acid (C14:1) is $\sim 14$ bonds long. Allowing each bond $1.33 \AA$ along the long molecular axis gives a total length of $19 \AA$, or $\sim 4 \mathrm{~nm}$ for a bilayer.

27. Cistola DP, Hamilton JA, Jackson D, Small DM. Biochemistry 1988;27:1881-1888. [PubMed: 3378036]

28. Mui BL, Cullis PR, Evans EA, Madden TD. Biophysical Journal 1993;64:443-453. [PubMed: 8457670]

29. Jin AJ, Huster D, Gawrisch K, Nossal R. European Biophysics Journal 1999;28:187-199. [PubMed: 10192934]

30. Struck DK, Pagano RE. J. Biol. Chem 1980;255:5404-5410. [PubMed: 7372642]

31. Gribbon P, Hardingham TE. Biophys. J 1998;75:1032-1039. [PubMed: 9675204]

32. Sanders CR, Prosser RS. Structure 1998;6:1227-1234. [PubMed: 9782059]

33. Israelachvili, JN. Intermolecular and Surface Forces. 2nd ed.. Academic Press; London: 1991.

34. Hub H-H, Hupfer B, Koch H, Ringsdorf H. Angew. Chem. Int. Ed. Engl 1980;19:938-940. [PubMed: 6779676]

35. Mueller A, O'Brien DF. Chem. Rev 2002;102:727-757. [PubMed: 11890755]

36. Regen SL, Shin J-S, Yamaguchi K. J. Am. Chem. Soc 1984;106:2446-2447.

37. Ruysschaert T, Germain M, Gomes JF, Fournier D, Sukhorukov GB, Meier W, Winterhalter M. IEEE Trans. Nanobioscience 2004;3:49-55. [PubMed: 15382644]

38. Ringsdorf H, Schlarb B, Tyminski PN, O'Brien DF. Macromolecules 1988;21:671-677.

39. Ringsdorf H, Schlarb B, Venzmer J. Angew. Chem. Int. Ed. Engl 1988;27:113-158. 


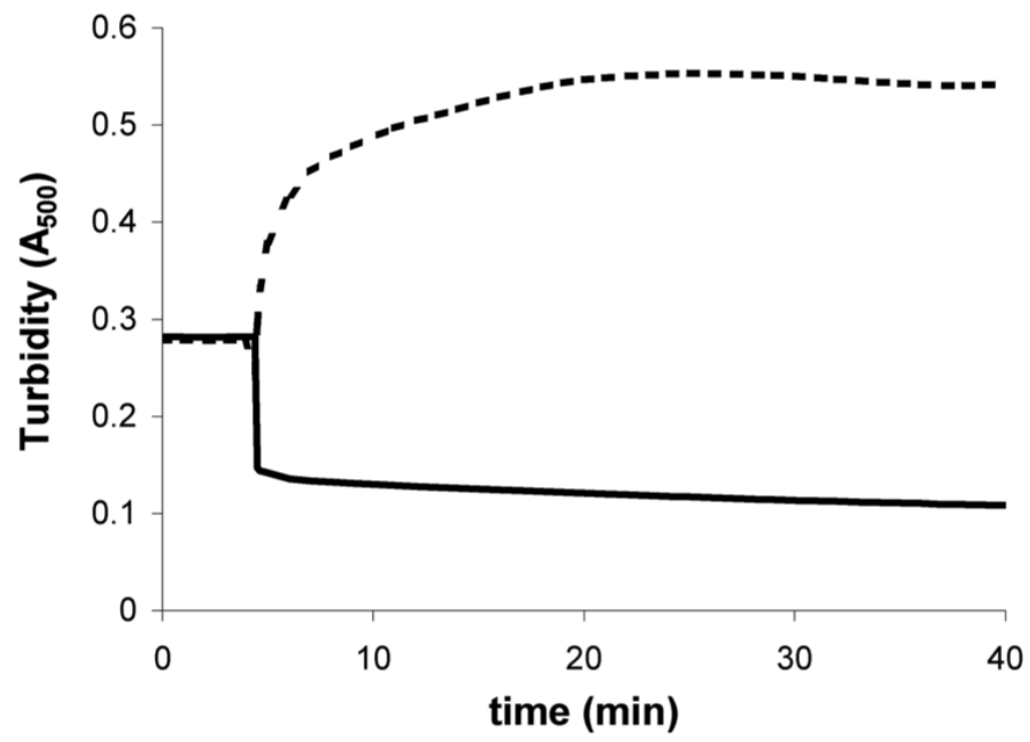

Figure 1.

Turbidity of MA vesicles after addition of MA/POPC (89:11) micelles (solid line) compared to addition of pure MA micelles (dotted line). The mixture contained $20 \mathrm{mM}$ vesicles and 25 $\mathrm{mM}$ micelles. Micelles were added at $t=4 \mathrm{~min}$. 

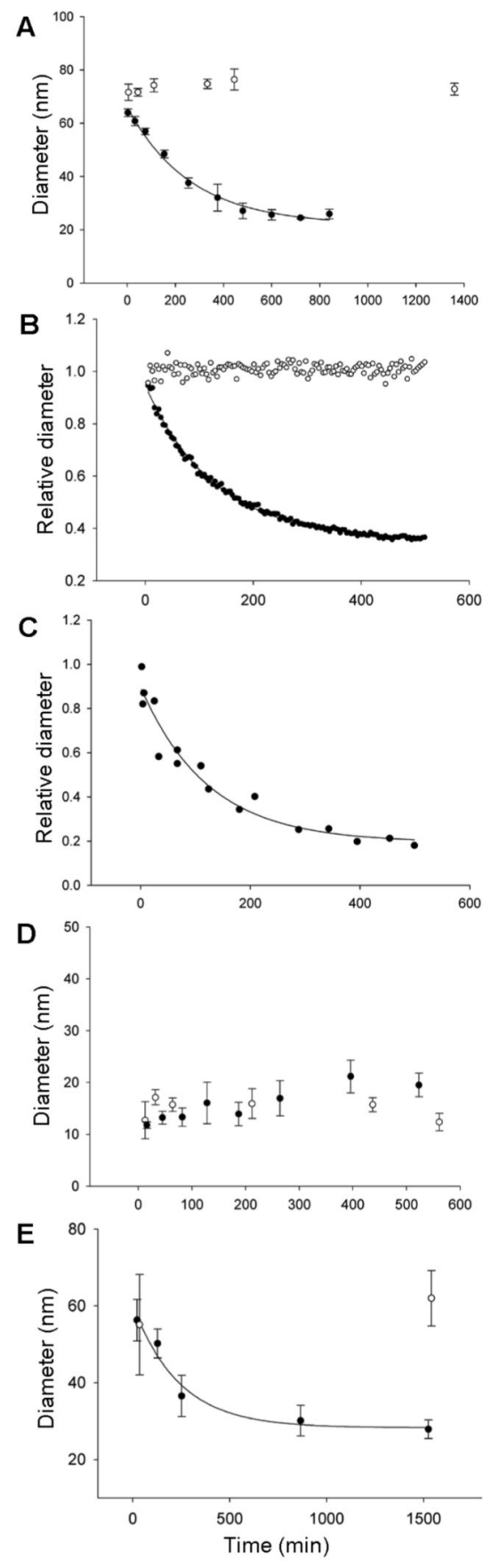

Figure 2.

Decrease in size of MA vesicles (3.2 mM, unless otherwise indicated) after mixing with MA/ POPC micelles (89\% MA, 11\% POPC; $4 \mathrm{mM}$ lipid, unless otherwise indicated). Solid lines show curve fits with parameters given in the text. $(A)$ Diameter measured by dynamic light scattering. Empty circles: vesicles alone; filled circles: mixture. $(B)$ Diameter relative to initial diameter, measured by FRET assay. Empty circles: vesicles alone; filled circles: mixture. $(C)$ Diameter relative to initial diameter, measured by the calcein self-quenching assay. Data were compiled from seven separate shrinking experiments with initial [calcein] $=0.2,2$, or $20 \mathrm{mM}$. Vesicles alone showed $<8 \%$ dye leakage and change in quenching efficiency after 22 hours. (D) Diameter of labeled MA/POPC micelles determined by FRAP. Empty circles: $40 \mathrm{mM}$ 
micelles in buffer only; filled circles: mixture (20 mM micelles and $32 \mathrm{mM}$ vesicles). Micelles in water have an apparent diameter of $8 \mathrm{~nm}$ by this assay. $(E)$ Diameter of vesicles determined by FRAP (final diameter $=28 \mathrm{~nm} ; k_{\mathrm{FRAP}}=0.004 \mathrm{~min}^{-1}$ ). Empty circles: $32 \mathrm{mM}$ vesicles alone; filled circles: mixture (20 $\mathrm{mM}$ micelles and $32 \mathrm{mM}$ vesicles). 

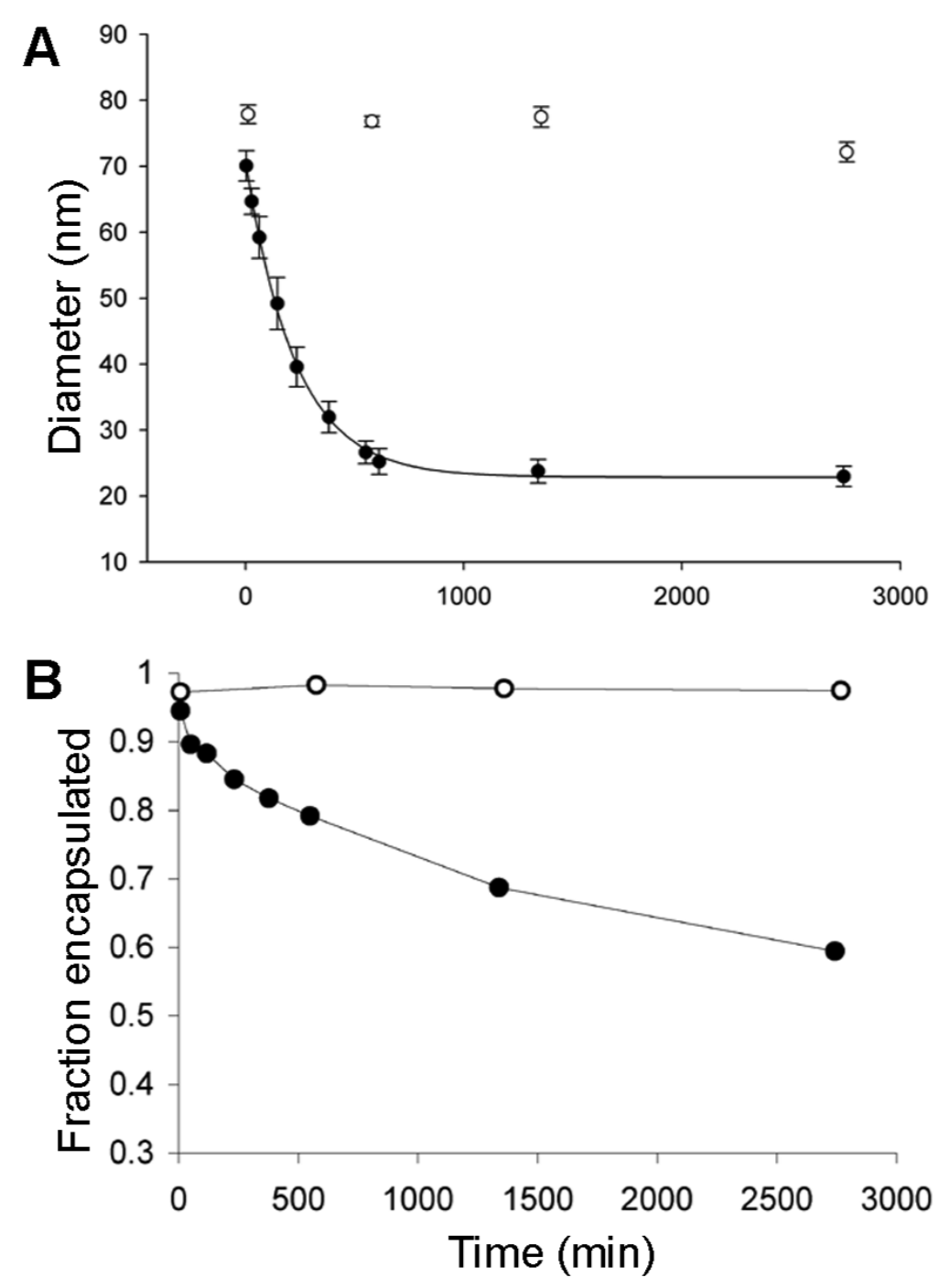

Figure 3.

Shrinking of MA vesicles initially containing $0.5 \mathrm{mM}$ HPTS. Filled circles: MA vesicles mixed with MA/POPC micelles; empty circles: vesicles alone. The average vesicle diameter decreased over time, as measured by DLS ( $A$; solid line shows a single exponential decay curve fit, with $k \sim 0.0044 \mathrm{~min}^{-1}$ and final diameter $\sim 23 \mathrm{~nm}$ ). Encapsulated HPTS was released slowly $(B)$, with a timescale much slower than the decrease of vesicle size. 

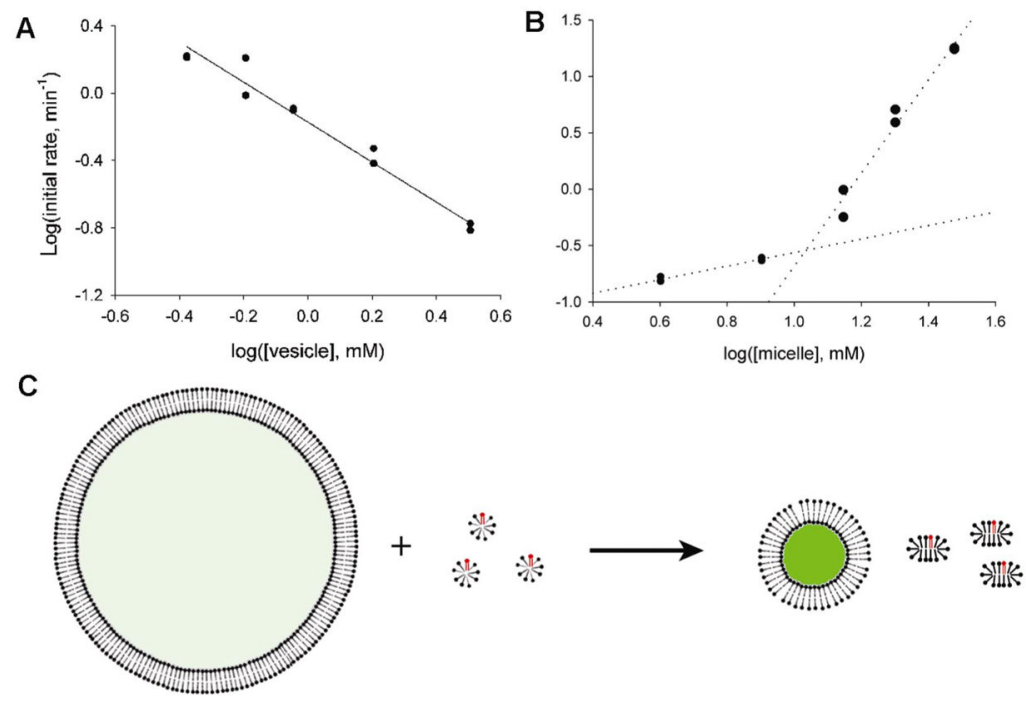

Figure 4.

Dependence of initial rate of vesicle shrinking on the concentration of reactants. (A) Van't Hoff plot indicating inhibition of the reaction rate by vesicles (slope $\sim-1$ ). (B) Van't Hoff plot showing the effect of micelles on initial rate. The dotted lines are meant to guide the eye (slope $\sim 0.6$ and 4 ), suggesting the presence of more than one reaction order. (C) Diagram of the transfer of MA (black) from vesicles encapsulating calcein (green) to micelles containing POPC (red). Transfer of MA to micelles might create a local membrane-like geometry, relieving strain in the micelles caused by the geometry of POPC. 
Table 1

Rate and extent of vesicle shrinking.

\begin{tabular}{|c|c|c|c|c|}
\hline technique & & $\begin{array}{c}\text { initial [calcein] } \\
(\mathbf{m M})\end{array}$ & $\begin{array}{c}\text { final outer } \\
\text { diameter }(\mathbf{n m})\end{array}$ & $k\left(\min ^{-1}\right)$ \\
\hline DLS & $\begin{array}{l}\text { control } \\
\operatorname{mix} \\
\operatorname{mix} \\
\operatorname{mix}\end{array}$ & $\begin{array}{c}- \\
- \\
0.2,2 \\
20\end{array}$ & $\begin{array}{l}73 \pm 2 \\
20 \pm 3 \\
20 \pm 2 \\
26 \pm 3\end{array}$ & $\begin{aligned}- & \\
0.0037 & \pm 0.0002 \\
0.006 & \pm 0.001 \\
0.007 & \pm 0.001\end{aligned}$ \\
\hline FRET & & - & $26 \pm 3$ & $0.0073 \pm 0.0004$ \\
\hline $\begin{array}{l}\text { calcein self- } \\
\text { quenching }\end{array}$ & & $0.2-20$ & $20^{* *}$ & 0.008 \\
\hline FRAP & & - & 28 & 0.004 \\
\hline
\end{tabular}

* Mixture: 3.2 mM MA vesicles + 4 mM MA/POPC micelles, except for FRAP experiment (32 mM MA vesicles + 20 mM MA/POPC micelles). Errors given are standard deviations.

$* *$

assuming a membrane thickness of $4 \mathrm{~nm}$ 